\title{
Prostate stereotactic body radiotherapy with simultaneous integrated boost: which is the best planning method?
}

\author{
Alison Tree ${ }^{1 *}$, Caroline Jones ${ }^{1}$, Aslam Sohaib ${ }^{1}$, Vincent Khoo ${ }^{1,2}$ and Nicholas van As $^{1}$
}

\begin{abstract}
Background: The delivery of a simultaneous integrated boost to the intra-prostatic tumour nodule may improve local control. The ability to deliver such treatments with hypofractionated SBRT was attempted using RapidArc (Varian Medical systems, Palo Alto, CA) and Multiplan (Accuray inc, Sunnyvale, CA).

Materials and methods: 15 patients with dominant prostate nodules had RapidArc and Multiplan plans created using a $5 \mathrm{~mm}$ isotropic margin, except $3 \mathrm{~mm}$ posteriorly, aiming to deliver $47.5 \mathrm{~Gy}$ in 5 fractions to the boost whilst treating the whole prostate to $36.25 \mathrm{~Gy}$ in 5 fractions. An additional RapidArc plan was created using an $8 \mathrm{~mm}$ isotropic margin, except $5 \mathrm{~mm}$ posteriorly, to account for lack of intrafraction tracking.

Results: Both RapidArc and Multiplan can produce clinically acceptable boost plans to a dose of 47.5 Gy in 5 fractions. The mean rectal doses were lower for RapidArc plans (D50 13.2 Gy vs 15.5 Gy) but the number of missed constraints was the same for both planning methods (11/75). When the margin was increased to $8 \mathrm{~mm} / 5 \mathrm{~mm}$ for the RapidArc plans to account for intrafraction motion, 37/75 constraints were missed.

Conclusions: RapidArc and Multiplan can produce clinically acceptable simultaneous integrated boost plans, but the mean rectal D50 and D20 with RapidArc are lower. If the margins are increased to account for intrafraction motion, the RapidArc plans exceed at least one dose constraint in 13/15 cases. Delivering a simultaneous boost with hypofractionation appears feasible, but requires small margins needing intrafraction motion tracking.
\end{abstract}

Keywords: Stereotactic body radiotherapy, Prostate cancer, Focal boost

\section{Introduction}

Dose escalation is known to improve biochemical control in prostate cancer [1-4] however at conventional fractionations, this is associated with an increase in toxicity [1-3]. Studies deriving the alpha-beta ratio for prostate cancer from low-dose rate brachytherapy and external beam treatments have suggested the alpha-beta ratio is possibly as low as $1.5 \mathrm{~Gy}$ [5-7]. It is hypothesized that we can exploit this unusual tumour characteristic to permit radiobiological dose escalation without increasing toxicity using fewer, larger doses of radiation (hypofractionation). Studies of moderate hypofractionation (up to 3.5 Gy per fraction) have shown toxicity which is, at worst, equivalent to standard fractionation [8-10] and

\footnotetext{
* Correspondence: alison.tree@rmh.nhs.uk

'Royal Marsden NHS Foundation Trust, Fulham Road, London SW3 6JJ, UK Full list of author information is available at the end of the article
}

there are some early suggestions of improved biochemical control $[9,10]$. Further data is eagerly awaited. Stereotactic body radiotherapy (SBRT) is an emerging technique for treating localized prostate cancer using 4-5 doses of 7 Gy or higher $(36.25$ Gy in 5 fractions is a commonly used dose). Results from both retrospective and prospective series show good biochemical outcomes in those with low and intermediate risk disease [11-14], which serves to reaffirm the concept of a low alpha/beta ratio for prostate cancer. Almost all the literature on prostate SBRT delivers these treatments on Cyberknife but there is increasing interest in using conventional linacs to deliver SBRT.

Studies of patterns of failure following conventionally fractionated external beam radiotherapy show that the area responsible for local recurrence is the dominant intraprostatic nodule in 89\% [15] - 100\% [16] of cases. By dose escalating the dominant nodule we should

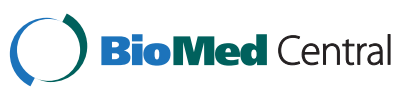


increase biochemical control whilst avoiding the increase in side effects seen with whole gland dose escalation.

SBRT using Cyberknife is capable of producing dose distributions that are similar to those achieved with HDR, with a rapid dose fall off and the ability to create heterogeneous dose distributions within the prostate [17]. The objective of this study is to achieve a heterogeneous dose distribution, with the high dose region (47.5 Gy) targeted to the dominant intraprostatic nodule, whilst maintaining a dose of 36.25 Gy to the whole prostate. We compared the two methods of SBRT delivery available to us at our institution - Cyberknife (using Multiplan planning software) and RapidArc arc therapy using Varian linacs.

\section{Materials and methods Patient selection}

This study was prospectively approved by the Royal Marsden service evaluation committee. Fifteen patients with intermediate or high risk prostate cancer, who had received standard IMRT at our institution, and who had a dominant prostate disease nodule (DPDN) on their diagnostic MRI, were selected. All patients had standard bladder filling protocol (300 mls at 45 minutes prior to planning CT scan) and no routine bowel preparation prior to the radiotherapy planning scan.

\section{Volume definition}

The diagnostic MRI scan was fused with the planning CT scan. The DPDN was contoured jointly by the oncologist and the radiologist using MRI (T2 and diffusion-weighted sequences were used to delineate the boost volume in all cases) and with reference to the sites of highest Gleason score described on pathology. The volume outlined formed the boost target volume.

The prostate and proximal seminal vesicles were outlined to form the CTV. Normal structures were delineated as per institutional protocol including delineation of the rectum as a solid structure extending from the anus to the recto-sigmoid flexure, and delineation of the bladder as a solid structure from bladder base to dome.

\section{Margins}

Our institutional margins for prostate SBRT using Cyberknife are $5 \mathrm{~mm}$ except $3 \mathrm{~mm}$ posteriorly. This is based on the literature on prostate SBRT $[11,12]$ and is concordant with those used in the current randomized PACE trial comparing SBRT with IMRT [18]. This margin is likely to account for subclinical extra-prostatic extension $[19,20]$ and treatment delivery uncertainties. The prostate and seminal vesicles CTV was therefore expanded by $5 \mathrm{~mm}$ isotropically except $3 \mathrm{~mm}$ posteriorly to form the PTV(prostate) for the Cyberknife plans.
For the RapidArc plans (which used RapidArc version 8.6), to provide pure dosimetric comparison, the first plan used the same margins ( $5 \mathrm{~mm} / 3 \mathrm{~mm}$ posteriorly) as we would use with Cyberknife to form the PTV(prostate). However, it is established that intrafraction motion during conventional fractionation requires a margin of 2-3 mm [21-24]. Therefore a second plan was constructed with PTV (prostate) margins of $8 \mathrm{~mm} / 5 \mathrm{~mm}$ posteriorly which is likely to be sufficient to account for intra-fraction motion after set-up to gold fiducial markers before treatment.

No PTV margin was put around the boost region which was always entirely contained within the prostate CTV. Whilst a $0 \mathrm{~mm}$ margin for treatment delivery accuracy is unlikely to be achievable, as the area surrounding the boost was within the CTV prostate, the dose fall off around the boost was relatively slow, and we noted that in most cases at $2 \mathrm{~mm}$ from the boost, the mean dose was maintained above 47 Gy. The boost volume (with no margin) is hereafter called PTV (boost).

\section{Dose}

Dose to the PTV(prostate) was 36.25 Gy in 5 fractions and dose to the PTV(boost) was 47.5 Gy. Dose schema is shown in Figure 1.

Plans for both RapidArc and Multiplan were normalized so that $\geq 95 \%$ of the target volume received $100 \%$ of the dose. The organ at risk (OAR) constraints were based on those currently used clinically at our institution (see Table 1) for prostate SBRT.

This planning study assumed that for Cyberknife plans treatment would be theoretically delivered with gold fiducial intra-fraction monitoring every 30-60 seconds. For RapidArc plans, it was assumed that patients would be set-up to gold fiducials prior to treatment.

Statistics were calculated using Prism version 6 (GraphPad software inc). Differences between planning methods were compared using a Wilcoxon matchedpairs signed rank test, as a Gaussian distribution could not be assumed and the correlation between $1 \mathrm{cc}$ dose and various parameters (PTV overlap with rectum, distance from boost to rectum, PTV volume) was explored using linear regression.

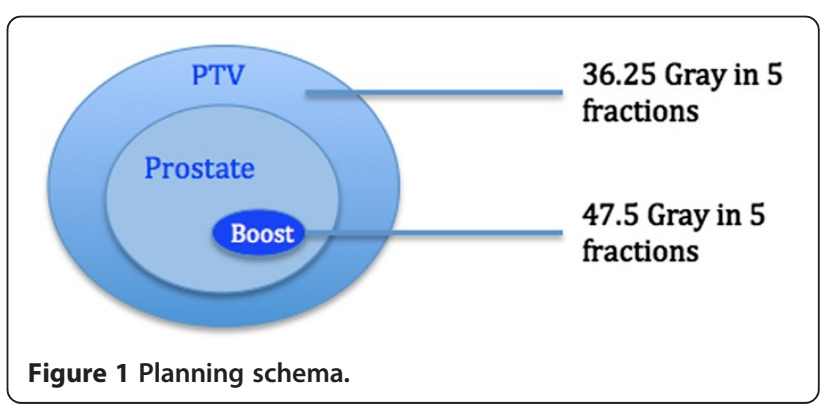


Table 1 Dose constraints for prostate SBRT planning

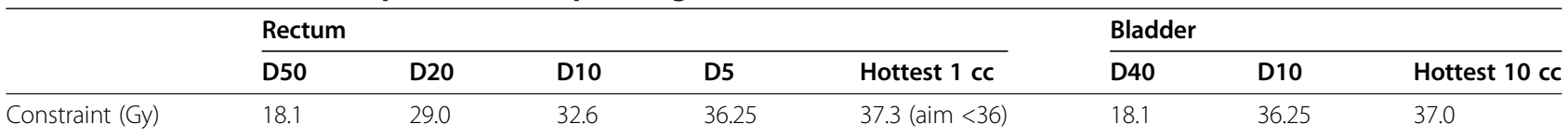

\section{Results}

\section{Plan demographics}

Patients had a mean age of 72, with a mean PSA of 19.1 and a mean prostate volume of $57.4 \mathrm{cc}$. Seven patients had T3a disease and the remainder had T2a-T2c. Due to the time, and in 13/15 cases the use of hormonal therapy between the diagnostic MR and the planning CT scan, and the inherent differences in CT and MR prostate outlining there was often significant change between the two scans which made fusion sub-optimal. In these cases, the prostate contour on the side of the boost was prioritized so that the boost region remained in a representative position. The boost was in the left lateral position in 6 cases, the right lateral position in 5 cases, was centrally positioned in 2 cases and was centrally posterior in 1 case. The mean boost volume was $3.7 \mathrm{cc}$ and the mean distance from the boost to the anterior rectal wall was $2.3 \mathrm{~mm}$. The mean overlap between the PTV $(5 \mathrm{~mm} /$ $3 \mathrm{~mm}$ margin) and the rectum was $2 \mathrm{cc}$ (range 0.02-4.26).

For Cyberknife plans the mean number of beams was 215 and the mean number of nodes was 59 . The mean predicted treatment time was 46 minutes and the mean conformality index for the Cyberknife plans was 1.14. For RapidArc, the plans were constructed with a double arc, and took at mean of 5.9 minutes to deliver and had a mean conformality index of 1.04 (smaller margins) and 1.03 (larger margins). The median boost dose was 47.5 Gy for both planning methods but the mean D95 PTV(boost) was higher for Cyberknife (48.1 Gy) than RapidArc (47.4 Gy) and RapidArc with larger margins (46.9 Gy).

\section{Rectal doses}

For the same margins, the mean doses to the rectum were higher on average for Multiplan (Figure 2 and Table 2) which may be partly a reflection of the way in which the optimization is driven in each system (e.g. we have found that with Version 8.6 of RapidArc the optimization is best if the OAR objectives are set much lower than the actual constraints, whereas with Multiplan over-reaching the possible OAR constraints can result in the degradation of the plan). In contrast, the number of constraints achieved vs failed was identical for Multiplan vs RapidArc with the same margins (see Figure 3). However for most cases where the constraint was failed, it was largely by less than $1 \mathrm{~Gy}$. For example for the 12 plans which could not meet the D10 constraint of $32.6 \mathrm{~Gy}, 8$ of achieved a D10 of 33.6 Gy i.e. only a 1 Gy relaxation of the constraint was needed for most plans to accomplish this. The constraint which was exceeded to the greatest extent was the rectal $1 \mathrm{cc}$ dose (i.e. dose to the hottest $1 \mathrm{cc}$ of rectum), which was in four cases $>2$ Gy higher than the constraint (2 Rapid Arc plans, 2 Cyberknife plans).

If the RapidArc margins were increased to $8 \mathrm{~mm} /$ $5 \mathrm{~mm}$ posteriorly then $43 / 75$ rectal constraints were missed. For example, the mean rectal D5 was 36.6 Gy for these plans (which is above the constraint of $36.2 \mathrm{~Gy}$ ) and the mean rectal $1 \mathrm{cc}$ was $37.7 \mathrm{~Gy}$, (maximum constraint $37.3 \mathrm{~Gy}$ ).

\section{Bladder doses}

For the bladder constraints, Cyberknife plans had higher mean values for the D40, D10 and hottest 1 cc compared to the RapidArc plans with the same margins. For example the mean dose to the hottest $10 \mathrm{cc}$ was 34.97 Gy for RapidArc and 35.88 Gy for Cyberknife. However, both of these are well within the constraint of 37 Gy (see Figure 4) and the number of missed constraints was similar.

The RapidArc plans with the larger margin gave more dose to all bladder constraints tested but they still met most constraints except the average dose to the hottest 10 cc of bladder which was 37.21 Gy, just outside the constraint. The association of bladder dose and genitourinary toxicity has not been well established with conventional fractionation and the implication of these dose differences in SBRT is not known.

\section{Are there factors which make focal dose escalation easier?}

As the constraint to the hottest $1 \mathrm{cc}$ of rectum was the hardest to meet, we examined whether certain factors are predictive of the value of this constraint. We investigated whether the extent of overlap between the PTV

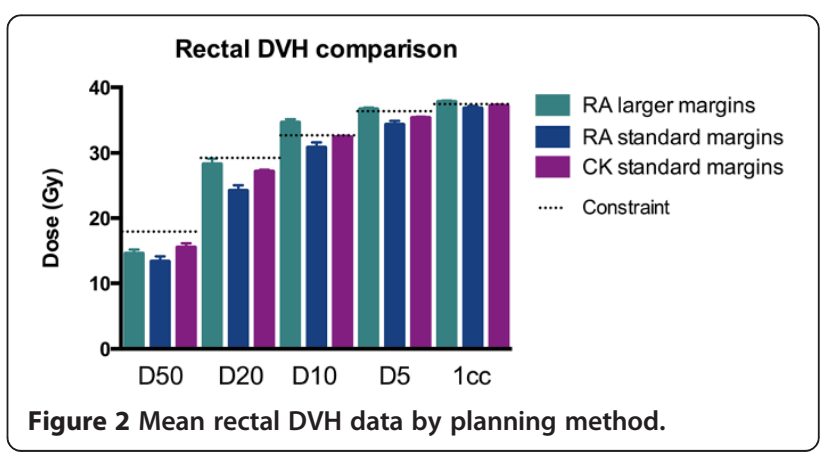


Table 2 Rectal dose comparison (figures in bold have exceeded the constraint)

\begin{tabular}{lllllll}
\hline Rectal dose & Margins & D50 & D20 & D10 & D5 & Hottest 1 cc \\
\hline Multiplan & $5 \mathrm{~mm} / 3 \mathrm{~mm}$ post & 15.52 & 27.13 & 32.40 & 35.32 & 37.15 \\
RapidArc & $5 \mathrm{~mm} / 3 \mathrm{~mm}$ post & $13.17^{*}(p=0.01)$ & $24.37^{*}(p=0.005)$ & 30.86 & 34.29 & 36.78 \\
RapidArc larger margins & $8 \mathrm{~mm} / 5 \mathrm{~mm}$ post & 14.59 & 28.28 & $\mathbf{3 4 . 6 1 *}(p=0.001)$ & $\mathbf{3 6 . 6 3 *}(p=0.0003)$ & $\mathbf{3 7 . 7}$ \\
\hline
\end{tabular}

*denotes those that are significantly different from Cyberknife plans (using a paired $t$-test, $\mathrm{p}$ values in brackets).

and the rectum, or the distance from the boost to the anterior rectal wall affected the ability to meet the most difficult constraint, the $1 \mathrm{cc}$ rectum dose.

Surprisingly, neither of these factors are clearly correlated with the dose to the $1 \mathrm{cc}$ rectum, except for the relationship between the Rectum-PTV overlap and the results of the RapidArc plans $(p=0.025)$. However this relationship loses statistical significance if the outlier (at $16 \mathrm{~mm}$ ) is removed.

We also examined for any correlation between dose to the hottest $1 \mathrm{cc}$ of the rectum and anterior-posterior, left-right patient separations at the level of the prostate and with prostate volume. No significant relationships were found.

\section{Discussion}

Many planning studies have compared the ability of different planning systems to deliver SBRT. This study compares the ability of Cyberknife and RapidArc to achieve an intentionally heterogeneous dose distribution and thereby achieve a differential dose to two PTVs depending on risk of disease recurrence.

There are many logical reasons why dose-escalating the dominant tumour nodule may improve the therapeutic ratio. However this strategy relies on being able to delineate the dominant prostate disease nodule accurately. Newer MRI techniques have improved tumour delineation in prostate cancer [25-27].

In the detection of patients with prostate cancer, combined dynamic contrast-enhanced (DCE) or diffusion weighted (DWI) MRI detects a cancer-containing region of the prostate with a high sensitivity and specificity.

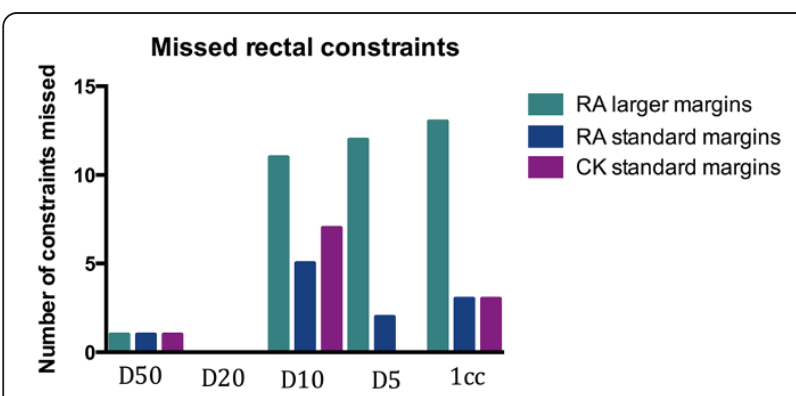

Figure 3 Number of missed rectal constraints by dose $(43 / 75$ for RapidArc larger margins, 11/75 for RapidArc standard margins, 11/75 for Cyberknife standard margins).
Studies have reported sensitivities of $38-82 \%$ and specificities of 37-96\% for DCE or DWI alone [25,26,28-30].

The combination of both DCE-MRI and DWI further improves delineation of tumour within the prostate [31-33]. The combination of DCE-MRI and MR-spectroscopy (MRS) may also improve tumour delineation [34]. It appears however that maximal tumour definition requires only 2 of the 3 modalities (DW-MRI, DCE-MRI and MRS), and that using all 3 does not improve prostate cancer detection [30].

Models which use MRI parameters (including DWI and DCE) on a voxel-by-voxel basis have been shown to be highly reliable for predicting tumour presence on pathology and suggested to be suitable for focal boost therapy [35]. The conspicuity of tumour nodules has been found to decrease in patients who have completed more than 3 months of androgen deprivation therapy (ADT) [36] and so it may be best to use a pre-ADT MRI to plan a focal boost, although the change in prostate size and shape over time may limit the accuracy of subsequent fusion to a radiotherapy planning CT. 18-F Choline-PET and Carbon-11 acetate PET have also been used to define a radiotherapy boost volume in planning studies $[37,38]$.

Studies have attempted to model the extent of focal dose escalation feasible with conventional fractionation. Housri et al. [39] identified DPDNs in 24/42 men studied. In these men dose escalation to $151 \mathrm{~Gy}$ was possible in half the cases, and was more likely to be possible in those with a larger distance between the dominant nodule and the rectum, and in those with a smaller hip-tohip distance on planning CT. This echoes our previously presented work, showing that DPDN within $1.5 \mathrm{~mm}$ of the rectum are more difficult to dose escalate [40].

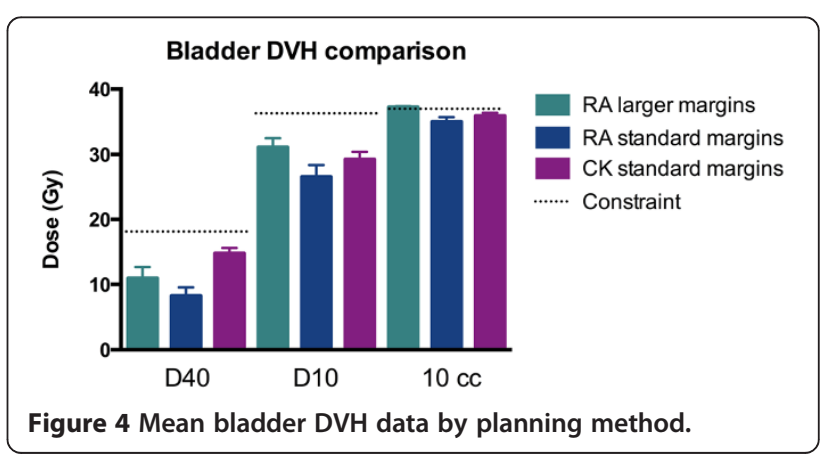


However, in this series, distance to rectum did not correlate with rectal $1 \mathrm{cc}$ dose, except for a finding of borderline significance for RapidArc plans. It may be that the ability to dose escalate to 47.5 Gy is correlated with distance to rectum, but that the dose to the $1 \mathrm{cc}$ rectum has other more complicated determinants, including possibly a combined effect of the size of the PTV, bladder and rectum and size of the patient.

Both RapidArc and Multiplan are complex planning tools and the quality of a plan is likely to be dependent on the experience of the user. Whilst the plans in this paper represent the best plans our department could produce at the time, we have had longer experience with RapidArc and hence our Cyberknife plans may still reflect the steep gradient of our learning curve.

It may also be that with further experience we have learnt to improve the dose fall-off of our plans, facilitating high intraprostatic doses with less penalty in rectal dose constraints. In addition, this is a sample of 15 patients, and larger numbers may be needed to see significant correlations.

The two systems discussed here have markedly different treatment times. The rate at which dose is delivered is likely to have some implication however the size and direction of this influence is complex [41] and likely to have a differential effect depending on $\mathrm{a} / \mathrm{b}$ ratio of the tissue in question. The oncological and side effect implications of a 40 minute vs a 6 minute treatment time, if any, are yet to be ascertained in vivo.

Both Multiplan and RapidArc planning systems can produce clinically acceptable plans which deliver a focal boost of 47.5 Gy in 5 fractions whilst treating the entire prostate to $36.25 \mathrm{~Gy}$. The extent of focal dose escalation possible without unacceptable OAR dose penalty may seem surprising but the use of either many beams, or arcing beams facilitates a rapid dose fall-off. The Cyberknife system incorporates near real-time tracking of the prostate which allows smaller margins to be used, such as those used in this study, as it tracks and corrects for intra-fraction motion. This paper suggests that if intra-fraction motion could be tracked and corrected during arc-therapy on a conventional linac, then the dosimetry is likely to be at least as good as Cyberknife. However, without intra-fraction motion control, a margin of $2-3 \mathrm{~mm}$ is likely to be needed to account for intrafraction motion after initial set-up to gold markers [42] which in this study is associated with exceeding dose constraints around half of the time. Alternatively, if new flattening-free filter linacs can deliver these plans in much shorter times, then intra-fraction motion may be less important, although transient, significant excursions of prostate position are still possible [43].

The optimization algorithms for RapidArc and Multiplan are different and, at least in our hands, these differences result in a slightly lower mean dose for RapidArc compared to Multiplan. In further work, not shown here, we have found that Multiplan can produce similar DVH values to RapidArc on individual plans, once the lowest achievable levels have been established with a RapidArc plan. It may be that further improvement in the Multiplan plans is possible as we gain further experience with this technique or as the optimization algorithm evolves.

We have established that it is possible to focally dose escalate prostate SBRT to target the dominant lesion. The next step is to establish if this can be achieved in patients without significant increase in toxicity, and whether focal dose escalation translates into improved biochemical control. This is being tested with conventional fractionation in the FLAME trial [44] which is currently randomizing patients (single-blind) to standard radiotherapy (77 Gy in 35 fractions) or the same dose plus a simultaneous integrated boost to the dominant nodule to $95 \mathrm{~Gy}$. This trial is defining the boost volume using MRI, including DCE- and DWI-MRI. The primary end-point is biochemical relapse-free survival at 5-years. Previous non-randomised studies have shown that moderate boosting to the dominant nodule (80-82 Gy) with conventional fractionation can be achieved without increase in toxicity [45-48].

The margins needed for an intra-prostatic boost are not well defined. Some studies have used $2-4 \mathrm{~mm}$ margins $[45,47,49]$ whereas others have not used an intraprostatic PTV margin [46]. As the boost is within the PTV (prostate) the dose fall-off is likely to be shallow enough to significantly dose-escalate $2-3 \mathrm{~mm}$ away from the delineated DPDN.

We have designed a pilot study of focal boosting using 5 fractions of SBRT, based on the planning technique used for this study. The SPARC trial (Stereotactic prostate augmented radiotherapy with Cyberknife) aims to establish if focal doses up to 47.5 Gy in 5 fractions (and 36.25 Gy in 5 fractions to the whole prostate) can be delivered to patients with intermediate and high risk prostate cancer without significant increase in acute toxicity.

\section{Conclusion}

The ability to utilize non-coplanar, non-isocentric beams did not significantly improve focal boost plans compared to arc therapy. Rapid Arc plans delivered lower mean rectal doses, but both systems achieved the dose constraints in the same number of cases. However, without the ability to track intrafraction motion the PTV would have to be increased by a further $2-3 \mathrm{~mm}$, particularly as the plans take an average of 5.9 minutes to deliver. If the PTV margin is increased to this extent, plans no longer meet the constraints in many cases. Intra-fraction motion monitoring facilitates the smaller margins needed to deliver a simultaneous integrated boost to the dominant 
prostate cancer nodule. We will shortly trial this technique clinically in a pilot study to assess whether these SIB plans can be delivered clinically without additional toxicity.

\section{Competing interests}

Dr. van As is the chief investigator of a randomised clinical trial which is financially supported by Accuray inc.

Dr. Tree, Dr. van As and Dr. Khoo have received educational support grants to facilitate attendance at international conferences.

The other authors declare no conflicts of interest.

\section{Authors' contributions}

NVA, VK and AT developed the concept and design of this study. AT, AS and CJ participated in the contouring and/or radiotherapy planning. All authors helped to draft and approved the final version of the manuscript.

\section{Acknowledgement}

We acknowledge support from the NIHR RM/ICR Biomedical Research Centre. The Royal Marsden NHS foundation trust is in receipt of an unrestricted educational grant from Accuray to support a research fellow.

\section{Author details}

'Royal Marsden NHS Foundation Trust, Fulham Road, London SW3 6JJ, UK. ${ }^{2}$ Institute of Cancer Research, Surrey SM2 5PT, UK.

Received: 9 July 2013 Accepted: 28 September 2013

Published: 2 October 2013

\section{References}

1. Al-Mamgani A, et al: Update of Dutch multicenter dose-escalation trial of radiotherapy for localized prostate cancer. Int I Radiat Oncol Biol Phys 2008, 72(4):980-8.

2. Dearnaley DP, et al: Escalated-dose versus standard-dose conformal radiotherapy in prostate cancer: first results from the MRC RT01 randomised controlled trial. Lancet Oncol 2007, 8(6):475-87.

3. Pollack $A$, et al: Prostate cancer radiation dose response: results of the $M$ D. Anderson phase III randomized trial. Int J Radiat Oncol Biol Phys 2002, 53(5):1097-105.

4. Zelefsky MJ, et al: Long-term results of conformal radiotherapy for prostate cancer: impact of dose escalation on biochemical tumor control and distant metastases-free survival outcomes. Int I Radiat Oncol Biol Phys 2008, 71(4):1028-33.

5. Brenner DJ, et al: Direct evidence that prostate tumors show high sensitivity to fractionation (low alpha/beta ratio), similar to lateresponding normal tissue. Int J Radiat Oncol Biol Phys 2002, 52(1):6-13.

6. Miralbell $\mathrm{R}$, et al: Dose-fractionation sensitivity of prostate cancer deduced from radiotherapy outcomes of 5,969 patients in seven international institutional datasets: alpha/beta $=1.4(0.9-2.2) \mathrm{Gy}$. Int J Radiat Oncol Biol Phys 2012, 82(1):e17-24.

7. Dasu A, Toma-Dasu I: Prostate alpha/beta revisited - an analysis of clinical results from 14168 patients. Acta Oncol 2012, 51(8):963-74.

8. Dearnaley D, et al: Conventional versus hypofractionated high-dose intensity-modulated radiotherapy for prostate cancer: preliminary safety results from the CHHiP randomised controlled trial. Lancet Oncol 2012, 13(1):43-54

9. Arcangeli $\mathrm{G}$, et al: A prospective phase III randomized trial of hypofractionation versus conventional fractionation in patients with high-risk prostate cancer. Int J Radiat Oncol Biol Phys 2010, 78(1):11-8.

10. Yeoh EE, et al: Hypofractionated versus conventionally fractionated radiotherapy for prostate carcinoma: final results of phase III randomized trial. Int J Radiat Oncol Biol Phys 2011, 81(5):1271-8.

11. Freeman DE, King CR: Stereotactic body radiotherapy for low-risk prostate cancer: five-year outcomes. Radiat Oncol 2011, 6:3.

12. Katz AJ, et al: Stereotactic body radiotherapy for organ-confined prostate cancer. BMC Urol 2010, 10:1.

13. King $C R$, et al: Stereotactic body radiotherapy for localized prostate cancer: interim results of a prospective phase II clinical trial. Int J Radiat Oncol Biol Phys 2009, 73(4):1043-8.
14. Meier $\mathrm{R}$, et al: Stereotactic body radiotherapy for intermediate-risk organconfined prostate cancer: interim toxicity and quality of life outcomes from a multi-institutional study. Int J Radiat Oncol Biol Phys 2012, 84(3):S148.

15. Arrayeh $\mathrm{E}$, et al: Does local recurrence of prostate cancer after radiation therapy occur at the site of primary tumor? Results of a longitudinal MRI and MRSI study. Int I Radiat Oncol Biol Phys 2012, 82(5):e787-93.

16. Cellini $\mathrm{N}$, et al: Analysis of intraprostatic failures in patients treated with hormonal therapy and radiotherapy: implications for conformal therapy planning. Int J Radiat Oncol Biol Phys 2002, 53(3):595-9.

17. Fuller DB, et al: Virtual HDR CyberKnife treatment for localized prostatic carcinoma: dosimetry comparison with HDR brachytherapy and preliminary clinical observations. Int J Radiat Oncol Biol Phys 2008, 70(5):1588-97.

18. The PACE trial. 2013. http://www.clinicaltrials.gov/ct2/show/NCT01584258.

19. Davis BJ, et al: The radial distance of extraprostatic extension of prostate carcinoma: implications for prostate brachytherapy. Cancer 1999, 85(12):2630-7

20. Chao KK, et al: Clinicopathologic analysis of extracapsular extension in prostate cancer: should the clinical target volume be expanded posterolaterally to account for microscopic extension? Int I Radiat Oncol Biol Phys 2006, 65(4):999-1007.

21. McNair HA et al: A comparison of the use of bony anatomy and internal markers for offline verification and an evaluation of the potential benefit of online and offline verification protocols for prostate radiotherapy. Int $J$ Radiat Oncol Biol Phys 2008, 71(1):41-50.

22. Adamson J, Wu Q: Inferences about prostate intrafraction motion from pre- and posttreatment volumetric imaging. Int I Radiat Oncol Biol Phys 2009, 75(1):260-7.

23. Beltran C, Herman MG, Davis BJ: Planning target margin calculations for prostate radiotherapy based on intrafraction and interfraction motion using four localization methods. Int J Radiat Oncol Biol Phys 2008, 70(1):289-95.

24. Kupelian P, et al: Multi-institutional clinical experience with the Calypso System in localization and continuous, real-time monitoring of the prostate gland during external radiotherapy. Int I Radiat Oncol Biol Phys 2007, 67(4):1088-98.

25. Schmuecking M, et al: Dynamic MRI and CAD vs. choline MRS: where is the detection level for a lesion characterisation in prostate cancer? Int $J$ Radiat Biol 2009, 85(9):814-24.

26. Kirkham AP, Emberton M, Allen C: How good is MRI at detecting and characterising cancer within the prostate? Eur Urol 2006, 50(6):1163-1174. discussion 1175

27. Turkbey B, Pinto PA, Choyke PL: Imaging techniques for prostate cancer: implications for focal therapy. Nat Rev Urol 2009, 6(4):191-203.

28. Testa C, et al: Prostate cancer: sextant localization with MR imaging, MR spectroscopy, and 11C-choline PET/CT. Radiology 2007, 244(3):797-806.

29. Tamada T, et al: Prostate cancer detection in patients with total serum prostate-specific antigen levels of 4-10 $\mathrm{ng} / \mathrm{mL}$ : diagnostic efficacy of diffusion-weighted imaging, dynamic contrast-enhanced MRI, and T2weighted imaging. AJR Am J Roentgenol 2011, 197(3):664-70,

30. Riches SF, et al: MRI in the detection of prostate cancer: combined apparent diffusion coefficient, metabolite ratio, and vascular parameters. AJR Am J Roentgenol 2009, 193(6):1583-91.

31. Kozlowski P, et al: Combined diffusion-weighted and dynamic contrastenhanced MRI for prostate cancer diagnosis-correlation with biopsy and histopathology. J Magn Reson Imaging 2006, 24(1):108-13.

32. Selnaes KM, et al: Peripheral zone prostate cancer localization by multiparametric magnetic resonance at $3 \mathrm{~T}$ : unbiased cancer identification by matching to histopathology. Invest Radiol 2012, 47(11):624-33.

33. Afaq $\mathrm{A}$, et al: Clinical utility of diffusion-weighted magnetic resonance imaging in prostate cancer. BJU Int 2011, 108(11):1716-22.

34. van Dorsten FA, et al: Combined quantitative dynamic contrast-enhanced MR imaging and (1)H MR spectroscopic imaging of human prostate cancer. J Magn Reson Imaging 2004, 20(2):279-87.

35. Groenendaal $\mathrm{G}$, et al: Pathologic validation of a model based on diffusion-weighted imaging and dynamic contrast-enhanced magnetic resonance imaging for tumor delineation in the prostate periphera zone. Int J Radiat Oncol Biol Phys 2012, 82(3):e537-44.

36. Groenendaal $\mathrm{G}$, et al: The effect of hormonal treatment on conspicuity of prostate cancer: implications for focal boosting radiotherapy. Radiother Oncol 2012, 103(2):233-8. 
37. Pinkawa $\mathrm{M}$, et al: Intensity-modulated radiotherapy for prostate cancer implementing molecular imaging with $18 \mathrm{~F}$-choline PET-CT to define a simultaneous integrated boost. Strahlenther Onkol 2010, 186(11):600-6.

38. Seppala J, et al: Carbon-11 acetate PET/CT based dose escalated IMRT in prostate cancer. Radiother Oncol 2009, 93(2):234-40.

39. Housri $\mathrm{N}$, et al: Parameters favorable to intraprostatic radiation dose escalation in men with localized prostate cancer. Int J Radiat Oncol Biol Phys 2011, 80(2):614-20.

40. Tree A, Khoo VS, van As N: To deliver a focal boost during whole prostate gland irradiation using Cyberknife. Radiother Oncol 2012, 103(s1):s494.

41. Ling CC, et al: Dose-rate effects in external beam radiotherapy redux. Radiother Oncol 2010, 95(3):261-8.

42. Litzenberg DW, et al: Influence of intrafraction motion on margins for prostate radiotherapy. Int J Radiat Oncol Biol Phys 2006, 65(2):548-53.

43. Langen $\mathrm{KM}$, et al: Observations on real-time prostate gland motion using electromagnetic tracking. Int J Radiat Oncol Biol Phys 2008, 71(4):1084-90.

44. Lips IM, et al: Single blind randomized phase III trial to investigate the benefit of a focal lesion ablative microboost in prostate cancer (FLAMEtrial): study protocol for a randomized controlled trial. Trials 2011, 12:255.

45. Fonteyne $V$, et al: Intensity-modulated radiotherapy as primary therapy for prostate cancer: report on acute toxicity after dose escalation with simultaneous integrated boost to intraprostatic lesion. Int J Radiat Oncol Biol Phys 2008, 72(3):799-807.

46. De Meerleer $\mathrm{G}$, et al: The magnetic resonance detected intraprostatic lesion in prostate cancer: planning and delivery of intensity-modulated radiotherapy. Radiother Oncol 2005, 75(3):325-33.

47. Pinkawa $M$, et al: Dose-escalation using intensity-modulated radiotherapy for prostate cancer - evaluation of quality of life with and without (18)Fcholine PET-CT detected simultaneous integrated boost. Radiat Oncol 2012, 7:14

48. Ippolito $E$, et al: Intensity-modulated radiotherapy with simultaneous integrated boost to dominant intraprostatic lesion: preliminary report on toxicity. Am J Clin Oncol 2012, 35(2):158-62.

49. Dose EscaLation to intraprostatic tumour nodules in localisEd prostATE cancer: A phase II study examining the toxicity and feasibility of a dose escalated boost to a magnetic resonance imaging identified tumour nodule or nodules in localised prostate cancer. [Accessed 9th September 2013]; Available from: http://www.controlled-trials.com/ISRCTN04483921.

doi:10.1186/1748-717X-8-228

Cite this article as: Tree et al:: Prostate stereotactic body radiotherapy with simultaneous integrated boost: which is the best planning method?. Radiation Oncology 2013 8:228.

\section{Submit your next manuscript to BioMed Central and take full advantage of:}

- Convenient online submission

- Thorough peer review

- No space constraints or color figure charges

- Immediate publication on acceptance

- Inclusion in PubMed, CAS, Scopus and Google Scholar

- Research which is freely available for redistribution 